die Tatsache des Vorhandenseins der Wasserstoffbombe in der Sowjetunion jene ziemlich einflussreichen Leute in den USA, die früher vom Präventivkrieg gesprochen haben, genötigt, ihre Zunge im Zaum zu halten. [...]

In dem weiteren Gespräch ging es um Fragen des Handels zwischen Ost und West, an dessen Ausweitung die UdSSR sehr interessiert war, und um Chruschtschows Lieblingsthema, die sowjetische Landwirtschaft, vor allem den Maisanbau.

Das Gespräch dauerte 1 Stunde und 35 Minuten

Übersetzt aus dem russischen Originaltext

RGANI, fond 52, opis' 1 , delo 552, Bl. 91-97

(Gesamter Text: B1. 87-101)

\title{
5. Gespräch mit dem amerikanischen Juristen Marshall McDuffie am 25. Januar 1956 (Auszug)
}

Zunächst unterhielten sich Chruschtschow und $\mathrm{McDuffie}{ }^{77}$ über unpolitische Dinge und sprachen danach über den Handel zwischen ihren Ländern, den Besuch amerikanischer Touristen in der UdSSR und den Kulturaustausch. Sie waren sich darüber einig, dass man diese Beziehungen verstärken müsse. McDuffie erzählte auch von Reisen und davon, dass er dabei in der Sowjetunion Fotos aufgenommen habe in der Absicht, dem amerikanischen Publikum das "wirkliche Leben“ dort zu zeigen. Weiter war in allgemeinen Wendungen von Chruschtschows Besuch in Indien, Burma und Afghanistan sowie vom Wohnungsbau, vom 6. Fünfjahrplan und von der Frauenkleidung in der UdSSR die Rede. Danach wurde das Gespräch wie folgt weitergeführt.

McDuffie: In Ihren Städten kann man mehr Soldaten und mehr Militärs auf den Straßen sehen als in anderen Ländern.

Chruschtschow: Was soll ich Ihnen darauf antworten, sagt Chruschtschow. Bei uns befinden sich alle Militärs im eigenen Land. ${ }^{78}$ Wir halten unsere Streitkräfte nicht außerhalb der Landesgrenzen, in Amerika dagegen befindet sich ein bedeutender Teil der Militärs außerhalb der Grenzen.79 Natürlich sehen Sie auf unseren Straßen Militärangehörige. Sie verstehen doch selbst, dass jeder Staat seine eigene Armee haben muss. Aber Sie wissen auch, dass wir das Kontingent unserer Streitkräfte erheblich reduziert haben. Dennoch haben wir eine Armee, und sie ist nicht klein.

Wir haben unsere Streitkräfte um 640000 Mann reduziert. Die Amerikaner und unsere anderen Partner haben unsere Vorschläge zur Reduzierung der Streit-

77 Marshall McDuffie war Professor der Rechtswissenschaft an der Harvard University. Als Linksliberaler stand er der Eisenhower-Administration kritisch gegenüber und hoffte auf ihre Ablösung durch die Demokraten. Der Sieg, den sie dann in den Wahlen vom November 1956 errang, war daher für ihn eine Enttäuschung.

78 Chruščëv ließ bei dieser Äußerung außer Betracht, dass sowjetische Truppen in der DDR sowie in Polen, Ungarn, Rumänien und Albanien stationiert waren.

79 Die Präsenz amerikanischer Streitkräfte in west- und südeuropäischen NATO-Staaten und in verschiedenen Ländern Südostasiens, namentlich in Japan, wurde den USA von der UdSSR als feindliche „imperialistische Einkreisung“ ständig zum Vorwurf gemacht. 
kräfte nicht angenommen, 80 sie haben auch die Vorschläge zur Abrüstung und zum Verbot der Atom- und Wasserstoffwaffen nicht angenommen. ${ }^{81}$

Gewisse amerikanische Kreise, merkt Chruschtschow an, haben Angst, den „Kalten Krieg“ zu liquidieren, weil sie damit Geschäfte machen, das heißt ihre Profite steigern, und das nicht schlecht. Die großen Monopole machen enorme Gewinne und jeder Monopolist streicht seine Prozente ein. Die einen stellen die Särge, die anderen die Kanonen her. Das eine wie das andere verkauft sich gut, und die, welche es herstellen, bekommen dafür Geld.

$M c D$ uffie: Auf diese Fragen möchte ich gern am Ende des Gesprächs zurückkommen.

Chruschtschow: Ich widerspreche Ihnen nicht. Weil Sie das Thema mit den Militärs angesprochen haben, habe ich Ihnen dazu ein paar schwarze Brocken hingeworfen (lacht).

$M c D u f f i e:$ Ich verstehe Sie, aber Sie haben mir viele derartige schwarze Brocken hingeworfen (lacht).

Chruschtschow: Welche Fragen also wollten Sie noch ansprechen?

McDuffie: Vielleicht ist es [nur] eine kleine Sache, aber ich möchte Ihnen Folgendes sagen. In der Zeit, als ich in der Sowjetunion gelebt und verschiedene Motive fotografiert habe, wurde ich zwei Mal von der Miliz ${ }^{82}$ angehalten: das eine Mal in Kiew auf dem Kreschtschatik ${ }^{83}$ und das zweite Mal bei der Neujahrsfeier im Kremlpalast. Es war irgendwie unangenehm, irgendwie peinlich, als ob ich etwas Verbotenes getan hätte. Natürlich ist mir in beiden Fällen nichts geschehen, und ich konnte weiter fotografieren. Aber wäre es nicht besser, der Miliz klar zu machen, dass sie fotografierende Personen nicht stören soll.

Chruschtschow: Offenbar hatten in beiden Fällen die Milizionäre den großen Wunsch verspürt, ihre Macht zu zeigen. Sie wussten, dass man fotografieren darf, wollten aber zeigen, dass sie Sie fragen können, was Sie denn da tun. Der Wunsch, eigene Macht zu zeigen, lässt sich vielfach auch nicht irgendwie durch Disziplin beseitigen. (McDuffie lacht)

Sie wissen doch selbst, dass man unmöglich jedes Verhalten aller Milizstreifen voraussehen und verhindern kann.

80 Am 12. August 1955 war auf Chruščëvs Betreiben hin eine Reduzierung der sowjetischen Streitkräfte um 350000 Mann bekannt gegeben worden. Am 17. Mai 1956 war von einer Verringerung um nochmals 420000 Mann die Rede, wovon freilich nur 300000 Soldaten zur Entlassung anstanden, weil es sich bei dem Rest um eine unerreichte, also nicht vorhandene Personalsollstärke handelte.

81 Die sowjetische Seite hatte im Zusammenhang damit die Forderung nach Abzug der fremden Truppen vom Gebiet der europäischen Staaten und nach Liquidierung der ausländischen Stützpunkte verbunden. Das war für die USA und ihre Verbündeten von vornherein unannehmbar, weil dies die Auflösung der Bündnisbeziehungen und das Ende des westeuropäischen Verteidigungssystems bedeutet hätte. Dahinter stand das Verlangen nach Aufbau eines Systems der kollektiven Sicherheit in Europa, das die UdSSR zur führenden Macht auf dem Kontinent machen sollte.

82 So hieß die Polizei in der UdSSR.

83 Hauptgeschäftszentrum von Kiev. 
$M c D$ uffie bemerkt, dass er in diesem Zusammenhang sich an seine Kindheit erinnert. Als er ein Junge war, hat er die einfache Sache kapiert, dass er, wenn er von einem Polizisten angehalten wurde, erihn am besten loswurde, indem er sagte: „Zu Befehl, Chef!“

Das war, sagt McDuffie, die beste Methode, einen Mann freundlich zu stimmen, der mir einen Verweis erteilen wollte.

Chruschtschow: Offenbar haben sich die Jungen überall so verhalten und machen es weiter so. (Beide lachen)

Wir bemühen uns, die Kultur aller Sowjetmenschen zu heben, darunter auch die Kultur der Mitarbeiter unserer Milizorgane.

McDuffie: In dieser Angelegenheit ist ein großer Fortschritt sichtbar. Als ich in der Sowjetunion zum ersten Mal war, wurde ich 14-mal wegen des Fotografierens angehalten, beim zweiten Besuch viermal und beim dritten Besuch nur zweimal.

Chruschtschow: Da sehen Sie, was für ein Fortschritt [erreicht worden ist]. Würden die Vereinigten Staaten bei der Verbesserung der Beziehungen mit uns die gleichen Fortschritte machen, wären wir in dieser Zeit weit vorangekommen. (Beide lachen)

McDuffie: Das würde ich mir auch von Herzen wünschen.

Jetzt, fährt er fort, einige Worte zu den Visa. In Ihren Stellungnahmen haben Sie mehrfach gesagt, dass es bei Ihnen hinsichtlich der Visa für Ausländer keine Hindernisse gibt. Mir ist so ein Beispiel bekannt. In der Sowjetunion waren zwei amerikanische Studenten, die ein Visum für 4 Wochen hatten, es war aber abgelaufen. Sie wollten noch vier Wochen bleiben, wussten aber nicht, ob dafür das Visum verlängert werden musste.

Chruschtschow bemerkt, wenn es Fälle der Verzögerung bei der Ausgabe der Visa gibt, dann zeugt das von gewissen Abnormitäten unserer Beziehungen zu den USA. Aber wir geben doch Visa allen Amerikanern, die in die Sowjetunion einreisen möchten, die amerikanischen Behörden dagegen geben keine Visa für unsere Sowjetbürger. Zum Beispiel durften sowjetische Straßenbauer nicht zum Kongress in die USA fahren. Das Außenministerium der USA hat den sowjetischen Ingenieuren nicht erlaubt, nach Amerika zu fahren. Und das ist kein Einzelfall. Über solche Fälle wurde viel in der Presse berichtet. ${ }^{84}$

McDuffie bemerkt, dass die Demokraten, wenn sie im nächsten Jahr an die Macht kommen, ${ }^{85}$ solche Dummheiten nicht [mehr] machen werden.

Chruschtschow: Man muss sich nur darüber wundern, wie töricht manche Leute im amerikanischen Außenministerium vorgehen. Wir haben zum Beispiel mit

84 Die amerikanische Gesetzgebung war restriktiv, wenn es um die Einreise sowjetischer Partei- und Staatsfunktionäre ging.

85 In den Präsidentenwahlen vom November 1957 würde es darum gehen, ob Eisenhower im Amt bleiben oder der Gegenkandidat der Demokraten siegen würde. Die Hoffnung McDuffies, dass Letzteres der Fall sein würde, wurde enttäuscht. 
Herrn [Charles] Hearst ${ }^{86}$ ausgemacht, dass sowjetische Vertreter $\mathrm{zu}$ ihm nach Amerika kommen, um mit ihm den Ankauf von Hybrid-Mais-Saatgut zu vereinbaren. Diese Leute wollten als Geschäftsleute nach Amerika reisen - erst anschauen, und dann das Nötige kaufen. Uns wollte man aber schikanieren, als wären wir irgendwelche unseriösen Leute. Das ist seitens der Amerikaner einfach nicht solide.

Schließlich haben schon immer, seit uralten Zeiten, die ausländischen Kaufleute in anderen Ländern hohes Ansehen genossen. Jetzt verhält man sich in den USA ganz anders, so als ob man fürchte, dass unsere Sowjetmenschen während ihres Aufenthalts in Amerika irgendwelche Geheimnisse des Maisanbaus in den USA entdecken. Sie können Herrn Hearst fragen und er wird Ihnen wahrscheinlich sagen, sagt Chruschtschow, dass unsere Wissenschaftler und führenden Betriebe beim Anbau guten Hybridsaatgutes in nichts hinter denen in Amerika zurückstehen. Hearst ist ein sehr geschäftstüchtiger, interessanter und offenbar ehrlicher Mann, und er kennt seine Sache. Mit ihm sich $\mathrm{zu}$ unterhalten, war interessant.

$M c D$ uffie: Ich kenne Herrn Hearst recht gut und stimme Ihrem Urteil über ihn $\mathrm{zu}$.

Auf die Maiskolben deutend, die auf N. S. Chruschtschows Tisch liegen, sagt McDuffie, dass man mit dem Blick auf seinem Tisch sogleich sehen kann, wofür er sich gerade am meisten interessiert. Vor 6 Jahren sah McDuffie auf N. S. Chruschtschows Tisch Baumaterialien, und jetzt sind auf dem Tisch viele Maiskolben.

McDuffie sagt, dass sich Chruschtschows Äußerung über Vorrechte für die Kaufleute wie eine Wiederholung eines alten kapitalistischen Dogmas anhört.

Chruschtschow: Das ist kein Dogma, sondern eine richtige Einstellung zur Verbesserungen der Handelsbeziehungen - das ist ein gutes Indiz.

$M c D u f f i e:$ Sind die von Ihnen geäußerten Prinzipien heutzutage von Bedeutung?

Chruschtschow: Zweifellos. Wir empfangen gerne Leute, die zu uns zur Anbahnung von Geschäftsbeziehungen mit der Sowjetunion kommen. Daher haben wir auch Herrn Hearst empfangen, und wir werden jeden anderen empfangen, der mit solchen Zielen zu uns kommt. Wir können Vertretern des Auslands verkaufen, was sie brauchen, und bei ihnen kaufen wir ein, was wir brauchen. Ich denke, dass solche Geschäftsbeziehungen keine schlechten Beziehungen sind.

McDuffie: Ich möchte Sie nicht mit meinen kritischen Bemerkungen belästigen.

Chruschtschow: Warum? Ich habe es gern, wenn Sie kritische Bemerkungen machen, ich meine berechtigte kritische Bemerkungen. Die haben uns nie beleidigt und beleidigen auch jetzt nicht.

McDuffie: Ich möchte Ihnen Folgendes sagen. Ich bin durch 11 Länder gereist. Nicht selten haben mich Bürger dieser Länder nach Hause eingeladen, doch in

86 Von Chruščëv hoch geschätzter führender Viehzüchter und Großfarmer in den USA. 
der Sowjetunion hat mich keiner auch nur ein einziges Mal nach Hause eingeladen. Vielleicht gibt es in dieser Frage irgendeine Regelung?

Chruschtschow: Nein. Es gibt in dieser Hinsicht keiner Regelungen. Jeder Sowjetmensch lädt zu sich nach Hause ein, wen er will. Vielleicht mochten die, mit denen Sie verhandelt haben, aus irgendwelchen Gründen Ihnen ihre Wohnungen nicht zeigen, aus der Sorge heraus, dass der Gast ein nicht objektives Urteil fällen würde. Vielleicht kennen sie Sie nicht gut genug, Herr McDuffie. Aber das alles sind meine Vermutungen.

McDuffie: Die Sache ist die, dass ich 1940 viel reisen musste. Ich war in Nordafrika, in der Schweiz, in England, Portugal, Frankreich und in anderen Ländern. Überall wurde ich freundlich empfangen, aber hier irgendwie offiziell. Ich kann nicht verstehen, wieso.

Chruschtschow: Ich denke, hier zeigen sich die gespannten Beziehungen zwischen unseren Ländern. Die Länder, die Sie genannt haben, sind anders als die Sowjetunion. Es ist zu berücksichtigen, dass die Sowjetmenschen ihren Stolz haben. Wenn einige Kreise in den USA versuchen, allen ihren Willen zu diktieren, wenn einige Amerikaner sich herausfordernd gegenüber der Sowjetunion verhalten, ziehen die Sowjetmenschen daraus entsprechende Schlüsse. Das ist der Grund, wieso die Sowjetmenschen eine gewisse Wachsamkeit an den Tag legen gegenüber vielen Leuten, die aus Amerika zu uns kommen.

McDuffie: Aber ich denke, ihnen ist klar, dass es einen Unterschied gibt zwischen den herrschenden Kreisen und dem Volk.

Chruschtschow: Natürlich ist ihnen das klar. Aber einfache Menschen aus den USA lässt man nicht oft in die Sowjetunion. Beispielsweise hat das US-Außenministerium aus einem bestimmten Grund Angst, Paul Robeson ins Ausland zu lassen, offenbar aus Sorge, dass er etwas Verbotenes singt. ${ }^{87}$ Doch die Stimme von Paul Robeson ist stark, und viele hören sie.

Das Außenministerium [der USA] lässt offenbar nur bestimmt eingestellte Menschen ins Ausland. Nehmen Sie die folgende Tatsache, sagt N. S. Chruschtschow weiter. Unter den amerikanischen Farmern, die zu uns kamen, waren auch solche, die kaum Mais vom Hanf unterschieden. Wir haben das gewusst, wir haben gewusst, dass einige Farmer keine normalen Farmer sind. Offenbar waren

87 Der Schwarzamerikaner Paul Robeson (1898-1976) war ein Sänger, Theater- und Filmschauspieler, der seine große Zeit in den dreißiger und vierziger Jahren des 20. Jahrhunderts hatte, danach jedoch im Zuge des Kalten Krieges wegen seiner politischen Haltung großen Anfeindungen und Schwierigkeiten ausgesetzt war. Während seines Aufenthalts in London von 1927 bis 1939 war er unter dem Einfluss der Labour-Politiker Stafford Cripps und Clement Attlee und des Kommunisten Harry Pollitt zum überzeugten Sozialisten geworden. Er las Werke von Marx, Engels, Lenin und Stalin im Original, wurde vom sowjetischen Botschafter mit den amtlichen Interpretationen bekannt gemacht und reiste zusammen mit seiner Frau 1934 in die UdSSR. Er kam zu der Überzeugung, dass dort eine wahrhaft menschliche Gesellschaft entstanden sei, in der vor allem die Gleichheit aller unabhängig von ihrer Hautfarbe verwirklicht werde. Ohne jemals Parteimitglied zu sein, trat er in der Offentlichkeit als Kommunist und Stalin-Sympathisant hervor. Das führte dazu, dass man ihm in den frühen fünfziger Jahren den Reisepass entzog, dass seine Schallplatten aus den Läden verschwanden und dass er auf Schwarze Listen gesetzt wurde mit der Folge einer weitgehenden Verhinderung öffentlicher Auftritte. 
gewisse amerikanische Organisationen genötigt, solche Leute zusammen mit den Farmern zu uns zu schicken. Na gut, sie sind bei uns gewesen, sie haben sich umgesehen. Das ist ihre Sache.

Aber leider kommen zu uns ins Land meistens solche Leute aus Amerika. Und ich habe Ihnen gesagt, dass die Sowjetmenschen ein sehr gutes Auge für diese Dinge haben.

McDuffie spricht davon, dass er sich nicht so gut in diesen Dingen auskennen kann. Als er den Auftrag erhielt, die Mission der UNRRA in der Ukraine zu leiten, 88 hat er zur Bedingung gemacht, dass nur Leute, die er persönlich kannte, Mitarbeiter dieser Mission wurden. Und trotzdem, fährt McDuffie fort, war unter ihnen ein Mensch wie Fisher, den er zwar persönlich kannte, aber, wie sich herausstellte, nicht gut genug.

Chruschtschow: Sie haben doch einmal Herrn Douglas ${ }^{89}$ Ihren großen Freund genannt. Herr Douglas hat unser Land besucht. Hier hat er gute Worte und einigermaßen gerechte Sachen von sich gegeben, aber als er in die USA zurückgekehrt war, begann er etwas völlig anderes zu sagen. Auf diese Weise wurden aus einem Douglas zwei - in der Sowjetunion war er der eine und im Ausland ein ganz anderer.

McDuffie: Douglas war einmal mein Lehrer. Ich habe für ihn um die Genehmigung seiner Einreise in die Sowjetunion gebeten.

Chruschtschow: Wir haben ihm diese genehmigt.

$M c D u f f i e:$ Aus eben diesem Grund mache ich Ihnen gegenüber meine kritische Bemerkungen, sage Ihnen offen, was bei Ihnen gut ist und was schlecht.

Chruschtschow: Wir warten auf die Zeit, wenn zu uns ins Land echte amerikanische Touristen kommen mit reiner und offener Seele und mit ehrlichen, aufrichtigen Absichten. Sie werden uns verstehen. Wir werden mit ihnen gute, ehrliche Beziehungen haben.

McDuffie: Das denke ich auch. Als Herr Douglas zurück in die USA kam, hatte ich mit ihm einen Streit. Er sagte beispielsweise, dass es in den östlichen Sowjetrepubliken in den Gerichten [nur] Russen gibt. Ich war damit nicht einverstanden und zeigte ihm ein Foto, das ich in Baku gemacht habe und auf dem eine Gerichtssitzung zu sehen ist. Unter den Richtern waren vier Nationalitäten vertreten. Douglas schaute auf das Foto und sagte, dass Baku noch nicht der Osten [der UdSSR] ist.

88 Die United Nations Relief and Rehabilitation Administration wurde auf Initiative der USA, der UdSSR, Großbritanniens und Chinas am 3. November 1943 als Hilfsorganisation zur Repatriierung der Displaced Persons (DPs), also der von den Gegnern, besonders den Deutschen, zurückgehaltenen Personen (wie vor allem Kriegsgefangene und Arbeitsverpflichtete) gegründet und nach Kriegsende der Organisation der Vereinten Nationen unterstellt worden. Die DPs wurden in Lagern verpflegt und betreut, die jeweils von einem Team geleitet wurden.

89 Paul Douglas, demokratisches Mitglied des amerikanischen Senats, dessen dezidiert liberale Haltung ihn oft eigene Wege gehen ließ. 
Chruschtschow sagt, dass er die Artikel von Herrn Douglas gelesen hat. Es sind dumme Artikel. Und die Verleumdung der Sowjetunion, die seine Artikel enthalten, fügt uns keinen Schaden zu. Die Errungenschaften der östlichen Sowjetrepubliken sind für alle offensichtlich. Wir sind stolz auf diese Republiken. Doch die Artikel von Douglas über diese Republiken vermitteln den Amerikanern ein Bild vom Leben und von den Menschen der östlichen Sowjetrepubliken, das in ein verzerrtes, falsches Licht gestellt wird. Das ist eine bedauerliche Tatsache. Die Hirngespinste von Douglas kann man mit den Bissen einer Fliege vergleichen, die auf dem Rücken eines Ochsen sitzt. Bewegt der Ochse den Schwanz - weg ist die Fliege. Und der Ochse geht weiter seinen Weg, ohne darauf zu achten, ob es die Fliege überhaupt gegeben hat oder nicht.

McDuffie (lacht): Gewöhnlich sprechen wir in solchen Fällen von Elefant und Fliege.

Ich habe noch zwei Fragen. Die erste Frage ist folgende. Ich möchte etwas mehr über Ihre Jugendjahre erfahren, als den offiziellen Angaben zu entnehmen ist.

Chruschtschow: Wozu, mit welchen Absichten? Sie wissen doch, je mehr sich die Menschen an ihre Jugend erinnern, desto mehr betonen sie das herannahende Alter. Aber wir haben keine Zeit zum Altwerden - es gibt [noch] viel zu tun. Sehen Sie, welch neuen Fünfjahrplan wir uns vorgenommen haben!

$M c D u f f i e:$ Ich bin mit Ihnen einverstanden. Aber ich möchte dennoch sehr gerne ein wenig mehr über Ihr Leben in den jungen Jahren erfahren. Sie waren doch Schafhirte, Schlosser, haben ein Studium an einer Arbeiterfakultät ${ }^{90}$ gemacht.

Chruschtschow: Das alles sind Angaben auf Fragebögen, und sie sind veröffentlicht.

McDuffie: Ja, das stimmt. Aber in den Fragebögen gemachen Angaben gibt es nicht besonders viele Details. Mir scheint, dass das Wissen über die Führer unserer Länder zur Annäherung unserer Völker beiträgt. Je mehr amerikanische die Leute in Amerika über die Führer des Sowjetstaates erfahren, desto besser wird es sein. Nach Ihrer Reise nach Indien, Birma und Afghanistan ${ }^{91}$ kennen die Menschen dieser Länder Sie jetzt sehr gut. Und das ist offenkundig gut so. Und eben deshalb möchte ich einige Details aus Ihrer Biografie erfahren. Haben Sie zum Beispiel Enkel?

Chruschtschow: Ich glaube, das sind alles Alltagsfragen. Leute in gesetztem Alter geben sich gerne der Erinnerung hin. Ich mag das nicht und habe außerdem keine Zeit dazu. Ich will [etwas] für mein Volk, für meine Partei schaffen, will arbeiten, ohne meine Kräfte zu schonen, ohne die Hände in den Schoß zu legen.

90 Die Arbeiterfakultäten waren im sozialistischen System spezielle Einrichtungen zur Fortbildung von Arbeitern.

91 Chruščëv und Bulganin besuchten vom 18. November bis zum 13. Dezember 1955 Indien, Burma, Afghanistan und zum Schluss nochmals Indien. 
$\mathrm{Na}$, und in Bezug auf meine Biografie kann ich sagen, dass dies eine ganz gewöhnliche Biografie ist. Sie fragen, ab wann ich in der Kommunistischen Partei bin? Seit 1918. Doch schließlich ist das alles schon veröffentlicht.

Sie fragen mich, ob ich Enkel habe. Ich habe Enkel, es gibt nicht wenige Enkel. Einer der Enkel studiert bereits an der Militärakademie, bereitet sich darauf vor, Ingenieur zu werden. Das ist der Sohn meines Sohnes, der an der Front gefallen ist. Ich habe eine Enkelin, die in die 10. Klasse geht. Es gibt noch zwei Enkel, die bis jetzt nur das Gehen gelernt haben. Wobei der eine auch schon laufen kann. Sie werden noch viel Brei essen müssen, bis sie erwachsen sind. Aber einer von ihnen hat bereits Ansprüche entwickelt.

Gerade heute, als ich zum Mittagessen nach Hause kam, sagte er zu mir: Los, Opa, ich werde auf dir reiten.

Nun gut, ich lasse ihn reiten.

Schließlich reiten die Enkel auf allen Großvätern, alle Großväter lassen die Enkel auf sich reiten. Und ich sage Ihnen, dies ist sehr angenehm für die Großväter wie für die Enkel. So eine Form der Belastung ist keine Ausbeutung des Menschen durch den Menschen. ${ }^{92}$ Wir erkennen sie an und nehmen sie gern auf uns.

$M c D$ uffie (lacht) bedankt sich für diese einfachen Worte.

Er fragt, welche Augenfarbe Chruschtschow hat. Die Zeitschrift „Time“ behauptet, Sie haben blaue Augen, „Newsweek“ schreibt, dass sie braun sind. Für Amerika liegt das Geheimnis des Kremls nicht in der Atombombe, sondern in der Frage, welche Augenfarbe Chruschtschow hat.

Chruschtschow: Gott weiß, was für Augen ich habe. Ich frage Sie: Welche Ziffern sind auf Ihrer Uhr, arabische oder römische. Aber schauen Sie nicht auf die Uhr. Die Hälfte der Menschen, die danach gefragt werden, täuscht sich gewöhnlich.

$M c D u f f i e:$ Ich bin mir jetzt auch unsicher, aber ich glaube, dass es arabische Ziffern auf dem Zifferblatt sind. (schaut hin) Ja, arabische.

Chruschtschow: Aber viele irren sich und geben damit Anlass zu sagen, dass die Uhr sozusagen diesen Leuten eigentlich nicht gehört, obwohl sie sie jahrelang tragen. So auch die Augenfarbe. Mitunter wird man gefragt - welche Augenfarbe hat Ihr Freund? Man überlegt - und kann es nicht genau sagen.

McDuffie: Kommen wir zurück auf Ihre Augenfarbe. Ich glaube, sie sind braun.

Chruschtschow: Mir bleibt nichts anderes übrig, als Ihnen zuzustimmen.

$M c D$ uffie: Erlauben Sie mir jetzt, eine so ernste Frage wie die der Verringerung der internationalen Spannung anzusprechen. In unserem letzten Gespräch haben wir darüber gesprochen, und das hat möglicherweise in gewissem Umfang dazu beigetragen, dass der „Geist von Genf“ entstanden ist.

Chruschtschow: Dazu kann ich Ihnen Folgendes sagen. Die sowjetische Regierung hat viel getan für die Festigung des Vertrauens zwischen den Ländern. Und

92 Mit der Formel, dass der Mensch den Menschen ausbeutet, beschreibt die marxistisch-leninistische Doktrin das Wesen des kapitalistischen Systems. 
wir sind der Meinung, sagt N. S. Chruschtschow, dass gegenwärtig Bedingungen von der Art entstanden sind, dass die Spannung in den internationalen Beziehungen abnehmen kann und soll. Ich und meine Freunde - Bulganin, Molotow, Shukow, die in Genf waren ${ }^{93}$ und mit den Vertretern der drei anderen Großmächte verhandelt haben - sind zu der Schlussfolgerung gelangt, dass unsere amerikanischen, englischen und französischen Partner mehrheitlich ebenfalls nach Frieden streben und keinen Krieg wollen. Insbesondere ist eine starke derartige Überzeugung bei uns hinsichtlich des Präsidenten der USA, Herrn Eisenhowers, entstanden. Ich denke, dass sowohl Herr Eden als auch Herr Faure, die ihre Länder vertreten haben, auch keinen Krieg wollen.

$\mathrm{Na}$, und in Bezug auf uns, die Vertreter der Sowjetunion, lässt sich Folgendes sagen. Wir sind immer für den Frieden eingetreten, tun das auch jetzt und bemühen uns in jeder Weise um eine Verringerung der internationalen Spannungen. Wir werden auch weiter in dieser Richtung tätig sein. Aber es gibt Kräfte in den westlichen Staaten, die sich unseren Bestrebungen entgegenstellen. Sie, diese Kräfte, organisieren verschiedene Blöcke von der Art der NATO. Sie wollen keine Abschwächung der Spannungen, sie wollen keine Liquidierung des „Kalten Krieges“. Solche Leute gibt es bedauerlicherweise am meisten unter den staatlichen Akteuren der USA. Ich spreche nicht vom amerikanischen Volk - das amerikanische Volk will keinen Krieg, es will Frieden. Aber einige staatlichen Akteure der USA möchten jetzt das hohe Spannungsniveau in den internationalen Beziehungen künstlich aufrechterhalten, denn andernfalls lässt sich keine militaristische Politik mit ihren Folgen durchführen.

Chruschtschow bemerkt, dass es jetzt im Bestand der Regierung sehr viele Vertreter der größten amerikanischen Monopole gibt. In der Geschichte der USA hat es bis jetzt keine andere Regierung gegeben, die den Willen der großen Monopole so offensichtlich vertreten hat wie die jetzige amerikanische Regierung.

Die großen Monopole, die mit der Herstellung militärischer Produkte Profit machen, fürchten eine Abschwächung der internationalen Spannung sehr. Sie fürchten den Anbruch einer Zeit, in der man die Produktion der Waffen nicht mehr braucht, die sie nicht nur an die amerikanische Armee, sondern auch an andere Länder liefern, die sich an den aggressiven Pakten wie NATO, SEATO, 94 Bagdad-Pakt ${ }^{95}$ und anderen beteiligen.

Die Aufrechterhaltung der internationalen Spannung gibt den herrschenden Kreisen der USA die Möglichkeit, der Bevölkerung hohe Steuern abzunehmen und die Menschen mit der Kriegsgefahr einzuschüchtern. Aber die Wahrheit vor

93 Vom 18. bis 23. Juli 1955 kamen die Staats- und Regierungschefs der Vier Mächte in Genf zu Beratungen über Deutschland und die Abrüstung zusammen. Sie erzielten keine Einigung, erteilten aber den Außenministern den Auftrag, die Erörterungen im Herbst wieder aufzunehmen. Auch dabei wurde keine Differenz überwunden.

94 Nachdem die USA, Frankreich, Großbritannien, Neuseeland, Australien, die Philippinen und Thailand am 8. September 1954 einen Bündnisvertrag abgeschlossen hatten, gründeten sie am 19. Februar 1955 die South East Asian Treaty Organisation (SEATO) mit dem Hauptquartier in der thailändischen Hauptstadt Bangkok.

95 Dem am 24. Februar 1955 zwischen dem NATO-Mitglied Türkei und dem Irak vereinbarten Bündnis hatten sich am 5. April Großbritannien, am 23. Mai Pakistan und am 3. November der Iran angeschlossen. Die USA beteiligten sich indirekt durch unterstützende Vereinbarungen. 
den Menschen zu verbergen, wird jetzt immer schwieriger. Die einfachen Leute, unter anderem auch in den USA, glauben nicht daran, dass die Sowjetunion den Krieg will. Daher wird auch es auch für die Monopolisten, die Waffenproduzenten, immer schwieriger. Die Menschen glauben nicht daran, dass es jetzt erforderlich ist, [noch] mehr Waffen zu produzieren, dass es erforderlich ist, angestrengt aufzurüsten.

Wir sind fest davon überzeugt, dass die Verfechter des Kampfes für den Frieden, alle ehrenhaften Menschen auf der Erde, wenn sie beharrlich für den Frieden kämpfen, keine Kriegsentfesselung zulassen werden. Aber dafür muss man beharrlich und konsequent kämpfen.

Wenn man jetzt auf die Stellungnahmen von solchen Militaristen blickt, wie es in den USA bedauerlicherweise nicht wenige gibt, und ihre heutigen und früheren Reden liest, kann man sich unschwer davon überzeugen, dass es ganz andere Reden sind als vor 2-3 Jahren. Wenn damals diese Leute freche Reden gehalten und offen zur Entfesselung eines Krieges aufgerufen haben, dann entschließen sich heute nur [noch] wenige dazu. Die Aufrufe zum Krieg finden heute in verschleierter Form statt.

Zeigt dies etwa nicht, dass das Volk der USA angefangen hat, das laufende Geschehen, die Bedeutung des Geschreis über eine Kriegsgefahr seitens der Sowjetunion besser zu begreifen und zu verstehen? Daher sehen sich solche Politiker [jetzt] genötigt, ihre Kriegsleidenschaft zu mäßigen. Sie nehmen auf die Stimmung der Völker und ihr gestiegenes Selbstbewusstsein Rücksicht.

Wir werden keine Mühe scheuen, sagt N. S. Chruschtschow, um mit unserer Aktivität, die auf die Abschwächung der internationalen Spannung gerichtet ist, die Kräfte des Friedens noch mehr zu stärken und die Tätigkeit der Kräfte abzuschwächen, die sich um die Entfesselung eines neuen Krieges bemühen. Die Völker der Welt wollen keinen Krieg. Wer sich um die Entfachung eines Krieges bemüht, muss sich jetzt umstellen, weil er begreift, dass das Volk damit nicht einverstanden ist und ihn entfernen könnte. Ich verstehe das Wort „entfernen“ in dem Sinne, dass das Volk solche Leute weder in den Kongress [der USA] noch in andere Organe der Staatsmacht wählen wird, es wird solche Leute nicht unterstützen.

Es ist doch nicht von ungefähr, dass [Joseph] McCarthy, ${ }^{96}$ bildlich gesprochen, das Maul gestopft wurde, ${ }^{97}$ und er jetzt seltener auftritt. Weswegen ist das

96 Joseph („Joe“) McCarthy (1908-1957) machte sich die Schocks zunutze, die im Herbst 1949 die amerikanische Öffentlichkeit erfassten, als die UdSSR im September ihre erste Atombombe zündete und am 1. Oktober die Proklamation der Chinesischen Volksrepublik in Peking den Sieg der Kommunisten über die - von den USA unterstützte - Bürgerkriegspartei ins Bewusstsein hoben. Als junger, noch wenig bekannter Politiker erklärte er, politisch links eingestellte Verschwörer im eigenen Land, vor allem im State Department, hätten durch heimliche Kollaboration mit den Kommunisten die Misere verursacht, und forderte, damit müsse Schluss sein. Damit zog er die öffentliche Aufmerksamkeit auf sich. Das Foreign Relations Committee des Senats sah sich im Februar 1950 zur Bildung eines Subcommittee on the Investigation of the Loyality of State Department Employees unter dem Vorsitz von Millard Tydings veranlasst. Dieses legte am 17. Juli 1950 einen Bericht vor mit dem Fazit, dass seine Verdächtigungen unbegründet seien. McCarthy setzte jedoch die Denunziationen fort. Seine Behauptungen erschienen einem breiten Publikum plausibel, als seit dem ausgehenden Frühjahr 1950 der Verrat amerikanisch-britischer Atomgeheimnisse an die UdSSR durch an deren Entdeckung beteiligte Wissenschaftler wie Klaus Fuchs, 
so? Eben deswegen, weil seine Stellungnahmen, seine maßlos absurden und törichten Äußerungen jetzt keine Sympathie [mehr] beim amerikanischen Volk finden.

McDuffie: Ich war als Jurist von Senator [Ralph] Flanders bei seinem Prozess gegen McCarthy dabei.

Chruschtschow: Wir treten ein für die Durchsetzung der Abrüstung, ein Verbot der Atom- und Wasserstoffwaffen und ein Verbot der Atom- und Wasserstoffwaffentests. Wir treten für alles ein, was die Beziehungen zwischen den Völkern festigt, was den Menschen hilft, sich gegenseitig mehr und besser kennen zu lernen und was die Beziehungen zwischen den Ländern festigt, statt sie zu verschärfen.

McDuffie: So denken auch viele Amerikaner. So denken die Mitglieder meiner demokratischen Partei. Aber es gibt eine Frage, die der Masse der Amerikaner Sorge bereitet. Sie und andere hochrangige sowjetische Politiker sagen oft, dass Sie für den Kommunismus kämpfen, dass der Kommunismus siegen wird, mit Gewalt siegen wird. Das macht den einfachen Amerikanern Sorge. Das amerikanische Volk möchte in Ruhe und Frieden leben.

Was Sie sagen, bemerkt N. S. Chruschtschow, ist ein falsches Verständnis, eine Entstellung des Gedankens vom Sieg des Kommunismus.

Wir glauben, dass die Gesellschaft der Zukunft eine kommunistische Gesellschaft sein wird. Die kommunistische Gesellschaft ist die beste Gesellschaft, denn alle ihre Mitglieder werden alle von der Menschheit geschaffenen Güter nutzen können. Nur Kranke oder Idioten können denken, dass sich der Kommunismus mittels Drohungen, mittels Einschüchterung durch Atom- und Wasserstoffwaffen aufbauen lässt. Die kommunistische Gesellschaft gründet auf der großartigen marxistisch-leninistischen Lehre. Diese Lehre muss man kennen und gut verstehen.

Die Leute, die in ihrem Land eine kommunistische Gesellschaft errichten wollen, müssen mit dieser Lehre einverstanden sein. Und wenn sie mit dieser Lehre

Julius und Ethel Rosenberg bekannt wurde. Bei den Wahlen in den USA vom November 1952, als Eisenhower Präsident wurde und die ihn unterstützenden Republikaner eine knappe Mehrheit in beiden Häusern des Kongresses gewannen, wurde McCarthy nicht nur in den Senat gewählt, sondern vermochte auch den Eindruck zu erwecken, er habe wesentlichen Anteil am Erfolg der Republikaner. Deren Führung gab ihm zum Dank den Vorsitz des - unwichtig erscheinenden Government Operations Committee, wo er vermeintlich wenig Schaden anrichten konnte. Er baute jedoch diese Position zum mächtigen Zentrum seiner auf Entlarvung ausgerichteten Tätigkeit aus, indem er das von ihm geleitete Senatskomitee als Tribüne der Anklage benutzte, die landesweit über das Fernsehen verbreitet wurde. Dadurch erlangte er lange Zeit großen Einfluss auf die öffentliche Meinung in den USA.

97 Seit langem stand der - zum liberalen Flügel der Republikaner gehörende - Senator Ralph Flanders den Aktivitäten McCarthys ablehnend gegenüber. Nach seiner Ansicht lenkten diese die Aufmerksamkeit von den wichtigen Feldern der weltweiten Auseinandersetzung mit dem Kommunismus $\mathrm{ab}$ und stifteten Verwirrung und Zwietracht im eigenen Land. Als immer deutlicher wurde, dass McCarthy den Beweis für die Anschuldigungen schuldig blieb, brachte Flanders am 11. June 1954 im Senat eine Resolution ein, in der ihm ungebührliches Verhalten vorwarf und seine Entfernung forderte. Dem wurde nach langer Diskussion am 2. Dezember 1954 mit 65 gegen 22 Stimmen entsprochen. 
nicht einverstanden sind, [wenn] sie in ihrem Land die kommunistische Gesellschaft bzw. deren erste Etappe - die sozialistische Gesellschaft - nicht errichten wollen, wie kann man sie dazu zwingen, dies zu tun?

Wir betrachten die Sache so, sagt N. S. Chruschtschow. Jedes Volk entscheidet selbst und wird entscheiden, auf welche Weise es sich entwickeln wird. Das Volk, und nur das Volk, entscheidet die Frage seiner Gesellschaftsstruktur. Wenn das amerikanische Volk bei sich den Kommunismus nicht aufbauen will, dann wird das niemand für die Amerikaner tun, niemand zwingt sie dazu, den Kommunismus aufzubauen.

Wenn wir sagen, dass der Kommunismus siegen wird, gehen wir von unserer Überzeugung aus, dass alle ehrlichen Menschen danach streben, dass nicht nur sie, sondern auch die anderen gut leben und in der Lage sind, die Früchte ihrer Arbeit zu ernten. In der sozialistischen, in der kommunistischen Gesellschaft ist der Mensch des Menschen Freund. In dieser Gesellschaft gibt es keine Ausbeutung des Menschen durch den Menschen, gibt es keine ausbeuterischen Klassen, die auf Kosten der Arbeit anderer Klassen leben. Ist das denn schlecht? Sie nennen das eine Phantasie, aber wir, die Sowjetmenschen, haben diese Phantasie zur Wirklichkeit gemacht, indem wir die sozialistische Gesellschaft errichtet haben und im ganzen Land die kommunistische Gesellschaft aufbauen. Wir glauben daran, dass wir die kommunistische Gesellschaft aufbauen werden und dass alle Menschen auf der Welt sehen werden, welche Vorteile der Kommunismus mit sich bringt, so wie sie [schon jetzt] die großen Vorteile des sozialistischen Systems sehen.

McDuffie: Wir Amerikaner haben das Gefühl, wenn Sie vom Kampf für den Kommunismus sprechen, darin liegt eine gewisse Aggression.

Chruschtschow: Dieses Verständnis, erläutert N. S. Chruschtschow, ist eine bewusste Verdrehung der Tatsachen. Unser Kampf für den Kommunismus bedeutet, dass wir zu Hause für die Überwindung aller Schwierigkeiten beim Aufbau der kommunistischen Gesellschaft kämpfen. Dadurch, dass wir zu Hause für den Kommunismus kämpfen, machen wir durch ein anschauliches Beispiel klar, dass auch andere Völker in ihren Ländern die sozialistische Gesellschaft errichten können.

Unser Kampf für den Kommunismus ist durchaus kein Krieg für den Kommunismus. Es wäre dumm, für den Kommunismus Krieg zu führen. Ich habe schon früher meine Vorstellungen zu dieser Frage zum Ausdruck gebracht. 98

Die Ideologen des Kapitalismus verdrehen bewusst unsere Worte über den Kampf für den Kommunismus, um einfachen Leuten einen Schreck vor dem Kommunismus einzujagen.

98 Diese Aussage entspricht der These der „friedlichen Koexistenz“, die von der sowjetischen Parteiführung kurze Zeit später - auf dem XX. Parteitag der KPdSU vom 14. bis 25. Februar $1956-$ zur ideologisch verbindlichen, langfristigen Strategie der Außenpolitik erklärt wurde. Danach war der Gebrauch bewaffneter Gewalt im Verhältnis „zwischen Staaten unterschiedlicher Gesellschaftsordnung“ zu vermeiden. Damit sollte die Möglichkeit eines Krieges zwischen den Machtblöcken von Ost und West ausgeschlossen werden, der - zumal wegen der dann drohenden nuklearen Eskalation - auf beiden Seiten eine unermessliche Vernichtung erwarten ließ. 
McDuffie: Viele verstehen es so, insofern Sie für den Kampf eintreten, dann muss irgendwer siegen. Wieso können Sie uns denn nicht in Ruhe lassen?

Chruschtschow: Ich habe Sie verstanden, bemerkt N. S. Chruschtschow. Sie sehen und verstehen die Sache in der Weise, dass wir gegen Sie für den Kommunismus in Amerika kämpfen wollen. Die Sache ist nicht so. Wenn wir sagen, wir führen den Kampf für den Aufbau der kommunistischen Gesellschaft in unserem Land, dann heißt dies, dass wir für die Überwindung der vor uns liegenden Schwierigkeiten kämpfen. Wir haben in unserem Land den Sozialismus errichtet, und alle Menschen in den anderen Ländern sehen jetzt, dass der Sozialismus in der Sowjetunion tatsächlich errichtet wurde . Jetzt sind wir dabei, in unserem Land die kommunistische Gesellschaft aufzubauen, und wir werden sie aufbauen. Diese Gesellschaft wird noch größeren Fortschritt bringen als die sozialistische Gesellschaft. Schon jetzt sieht man im Ausland, wie viel fortschrittlicher das System im Sowjetland ist verglichen mit dem kapitalistischen System. Das zeigen die Erfolge unseres Landes.

Wir wollen nicht den Weg des Krieges für den Kommunismus beschreiten, sondern den Weg des friedlichen Wettstreits der zwei Systeme: des sozialistischen und des kapitalistischen. Wir denken nicht an einen Krieg für den Kommunismus. Wir wollen keinen Krieg, und wir brauchen keinen Krieg. Wir kämpfen für den Kommunismus, indem wir alle Schwierigkeiten überwinden, die sich uns auf unserem Weg zu diesem großartigen Ziel in den Weg stellen.

Ich wiederhole nochmals, sagt Chruschtschow weiter, dass die Errichtung eines neuen gesellschaftlichen Systems im Lande, auch in den USA, eine innere Angelegenheit der Völker dieser Länder selbst ist. Und niemand wird den Völkern gegen ihren Willen von außen dieses System aufzwingen. Ich möchte Sie fragen: Können Sie oder irgendein anderer Amerikaner sich für Ihre Kinder oder Enkelkinder verbürgen, dass diese nicht den Wunsch haben werden, eine kommunistische Gesellschaft aufzubauen? Ich denke nicht. Die geschichtliche Erfahrung lehrt, dass Menschen aus den bürgerlichen Klassen nicht selten zu überzeugten Anhängern des Kommunismus oder anderer revolutionärer Strömungen werden. Beispielsweise hat im alten Russland so ein Politiker gelebt wie Kropotkin - er kam aus dem Hochadel. Der Zar selbst hütete seinerzeit den kleinen Kropotkin und sagte $\mathrm{zu}$ ihm, dass aus dem kleinen Jungen einmal ein guter Page werde. Der Zar hätte natürlich nie gedacht, dass dieser kleine Junge einmal ein Theoretiker des Anarchismus werden würde. ${ }^{99}$

Die bürgerliche Gesellschaft ist auf der Unterdrückung des Menschen durch den Menschen aufgebaut. In der kapitalistischen Gesellschaft gibt es keine Idee, die alle Mitglieder dieser Gesellschaft vereinigen würde. Der Kapitalismus, das ist ein Übel für die Werktätigen, aber die Werktätigen bilden in der kapitalistischen Gesellschaft die Mehrheit. Alles Übel geht unter im Kampf mit dem Guten.

99 Fürst Pëtr Alekseevič Kropotkin (1842-1921) war ein berühmter Anarchist, dem eine herrschaftsund gewaltfrei sich entwickelnde Menschheit vor Augen stand. Bei der Durchsetzung dieses Ziels lehnte er jeden Gewaltgebrauch ab. 
Ich füge hinzu: Meine Worte über den Kampf sind nicht als Kampf mit Hilfe der Waffen, mit Hilfe der Armee zu verstehen, sondern als Kampf der Ideen. Wir glauben, dass die Idee des Kommunismus siegen wird. Sie wird sich des Bewusstseins von immer neuen Millionen Menschen bemächtigen, so wie sie sich des Bewusstseins der Millionen Menschen in unserem Land und in den Ländern der Volksdemokratie bemächtigt hat. Das ist nicht der Kampf eines Volkes gegen ein anderes, sondern, wenn Sie so wollen, ein Kampf des Volkes im Volk. Die Befürworter einer Richtung kämpfen gegen die Befürworter der anderen Richtung.

Das Ende dieses Kampfes wird eintreten mit dem Sieg der kommunistischen Gesellschaft, wenn alle Menschen wirklich gleich und wirklich frei sein werden.

Die kapitalistische Idee oder, besser gesagt, die Ideen des kapitalistischen Staates, das ist die Ausbeutung der Menschen durch die Menschen, das sind Ideen des maximalen Profits. Sind das etwa Ideen, die begeistern? Nein, das sind Ideen des Raubes.

McDuffie: (nickt mit dem Kopf). Ich denke, dass Ihre Erläuterung uns eine klarere Vorstellung gibt von den schwierigen Fragen, die uns erregen.

In diesem Zusammenhang möchte ich Ihre Anschuldigung gegen uns, also die Amerikaner, ansprechen, dass wir von einer „Position der Stärke“ aus agieren. Als ich in der Ukraine war, hat man mir erzählt, dass General Tschujkow ${ }^{100}$ unlängst erklärte habe, die Sowjetunion habe die USA im Bereich der thermonuklearen Waffen überholt, die Sowjetunion habe nicht weniger Bomben als die USA.

Ist eine solche Stellungnahme nicht ebenfalls als eine Stellungnahme aus „Position der Stärke“ heraus ${ }^{101} \mathrm{zu}$ verstehen?

Chruschtschow: General Tschujkow, sagt N. S. Chruschtschow, ist ein sehr guter General, den ich seit langem kenne, schon von der Front von Stalingrad.102 Seine Worte sind offenkundig so zu verstehen, dass wir in der Lage sein müssen, diejenigen abzuwehren, die uns angreifen wollen. Schließlich sagen doch bei Ihnen in den USA viele Generäle und Nicht-Generäle, dass sie dieses und jenes auf die Sowjetunion loslassen werden. Davon reden Radford ${ }^{103}$, Dulles ${ }^{104}$ und andere, sie drohen uns mit Atom- und Wasserstoffbomben.

100 Vasilij Ivanovič Čujkov, der von 1949 bis 1953 als Oberbefehlshaber der Gruppe der Sowjetischen Streitkräfte in Deutschland und Oberster Chef der Sowjetischen Kontrollkommission in der DDR eine herausragende Position inne gehabt hatte, wurde anschließend als Chef des Kiever Militärbezirks. Seit 1952 war er Kandidat des Zentralkomitees der KPdSU. 1955 wurde ihm der Rang eines Marschalls der Sowjetunion verliehen.

101 McDuffie bezog sich damit auf den sowjetischen Vorwurf an die Adresse der USA und der NATO, eine Politik „aus der „Position der Stärke“ heraus zu betreiben.

102 Chruščëv war 1942/43 als leitender Parteifunktionär an der Front von Stalingrad tätig.

103 Admiral Arthur Radford war Vorsitzender der Vereinigten Stabschefs der USA, der Befehlszentrale der amerikanischen Streitkräfte. Nach dem Amtsantritt der Eisenhower-Administration leitete er mit dem Argument, dass die Verteidigungsausgaben auf diese Weise effizienter einzusetzen seien („Mehr Knall für den Dollar“) und dementsprechend verringert werden könnten, die Nuklearisierung der Militärstrategie ein.

104 John Foster Dulles war seit dem Amtsantritt der Eisenhower-Administration im Januar 1953 amerikanischer Außenminister. Er verfolgte mit Nachdruck einen scharfen Kurs gegenüber den Kommunisten und der UdSSR. 
Und das bestätigt meine Worte, dass Drohungen von beiden Seiten zu nichts Gutem führen können. Man sollte nicht mit seiner Stärke drohen und sich nicht seiner Vorräte an Atom- und Wasserstoffbomben rühmen.

Über eben dieses Thema habe ich heute schon mit dem englischen Korrespondenten Graham Stanford gesprochen. Wenn wir uns gegenseitig bedrohen, entsteht ein Teufelskreis, sodass wir wie auch die andere Seite eine Politik aus der „Position der Stärke“ heraus betreiben. Und das ist eine gefährliche Politik, bemerkt N. S. Chruschtschow, die üblicherweise zum Krieg führt.

Daher muss man, wenn man keinen Krieg will, eine andere Politik betreiben; eine Politik aus der „Position der Stärke“ ist eine unkluge und vor allem eine kurzsichtige Politik. Und wir reagieren darauf mit der Erklärung, dass wir nicht schwächer sind als unsere möglichen Gegner, und wir reagieren darauf nur deswegen, weil uns eine solche Politik aufgezwungen wird. Aber wir verurteilen eine Politik aus der „Position der Stärke“ heraus und wenden uns entschieden dagegen.

McDuffie: Wie haben Sie die Erklärung von Dulles in der Zeitschrift „Life“105 aufgenommen?

Chruschtschow: Die Amerikaner selbst haben ihr Urteil darüber abgegeben, als sie in etwa erklärten, der Mann habe einen extremen Punkt erreicht, bemerkt $\mathrm{N}$. S. Chruschtschow. Jetzt möchte sich Herr Dulles selbst aus der Lage herauswinden, in die er geraten ist. Und als ich Herrn Eisenhower nach dieser Erklärung des Außenministers gefragt habe, hat dieser nicht zufällig gesagt, dass er diese Erklärung nicht gelesen hat.

Das ist der beste Ausweg für den Präsidenten aus so einer Lage: Er liest nicht, was sein Außenminister sagt. Ich denke, wenn Herr Eisenhower diese Erklärung gelesen hätte, wäre er über das, was Dulles gesagt hat, nicht erfreut gewesen.

McDuffie sagt, dass er trotzdem denkt, Präsident Eisenhower den Artikel von Dulles [tatsächlich] nicht gelesen hat.

Chruschtschow: Ich möchte dem nicht widersprechen, sagt N. S. Chruschtschow. Es ist durchaus möglich, dass Herr Eisenhower diesen Artikel nicht gelesen hat. Ich bezweifle keineswegs die Worte von Herrn Eisenhower. Ich habe eine sehr hohe Meinung von Herrn Eisenhower, dem Präsidenten der USA - das ist ein sehr anständiger Mensch und Staatsakteur. Er hält sein Wort. Das wissen wir

105 Dulles hatte am 13. Januar 1956 in einem Interview mit dem Chef des Washingtoner Büros von Time-Life, James Shepley, kritische Geschehnisse des Konflikts mit dem Kommunismus in Asien während des vergangenen Jahres angesprochen - die Bedrohung der direkt vor dem Festland gelegenen, aber zu Taiwan gehörenden Inseln Quemoy und Matsu durch China im April, die ebenfalls von China verursachte Unterbrechung der in Korea geführten Waffenstillstandsverhandlungen im Juni und die Belagerung und Einnahme von Dien Bien Phu durch den kommunistischen Vietminh im Frühjahr - und betont, die USA seien dabei bis an die Schwelle des Krieges gegangen. Die Fähigkeit, bis an diese Schwelle zu gehen, aber nicht zum Krieg zu schreiten, sei die notwendige Kunst. Wenn man davonlaufe und sich fürchte, sei man verloren. Die Vereinigten Staaten seien bis an die Schwelle gegangen und hätten der Gefahr ins Auge geblickt. Die letzte Verantwortung liege bei Präsident Eisenhower. Dieser sei bei keiner Gelegenheit zurückgewichen. Das Interview war am 16. Januar 1956 im Magazin „Life“ unter der Überschrift „How Dulles Averted War“veröffentlicht worden. 
noch aus dem Krieg, den wir gemeinsam gegen Hitler-Deutschland geführt haben. ${ }^{106}$

McDuffie: Dulles hat in seinem Artikel gesagt, dass wir einige Male am Rande des Krieges gestanden haben. Die Amerikaner glauben das nicht.

Chruschtschow: Jeder hat seine Vorstellung darüber, wo sich der Rand befindet, erwidert N. S. Chruschtschow. Ich kann schwer beurteilen, was Herr Dulles als diesen Rand versteht. Schließlich hat er das erklärt, und er ist der Außenminister der USA und weiß offenbar, ob sich Amerika am Rand des Krieges befunden hat, ob es Krieg anfangen wollte. Ich habe keine Beweise, die gegen diese Behauptung sprechen.

Aber ich kann sagen, sagt N. S. Chruschtschow weiter, wer Krieg anfängt, nimmt gegen das Volk Stellung.

Ich habe keine Daten zur Hand, um Ihnen zu sagen, Dulles habe die Menschheit nicht in einen Krieg stürzen wollen. Ich kann nur die Bemerkung machen, dass sich das, wovon Dulles gesprochen hat, unschwer machen lässt, wenn man einen so hohen Posten inne hat wie den des [amerikanischen] Außenministers.

$M c D$ uffie sagt, dass er der Erklärung von Dulles keinen Glauben schenkt, so wie ihr auch viele [andere] Amerikaner keinen Glauben schenken.

Chruschtschow: Ich wiederhole, dass ich keinen Grund habe, Dulles keinen Glauben zu schenken, bemerkt N. S. Chruschtschow. Wir als Politiker müssen daraus den Schluss ziehen, dass Politiker wie Dulles offenbar zu jedem Irrsinn bereit sind. Schließlich geht es hier um Krieg und überdies um einen nicht gewöhnlichen Krieg.

Fragen von Krieg und Frieden sind keine Fragen eines Spiels, sondern Fragen des Schicksals der Völker. Deswegen nahmen wir Dulles‘ Erklärung sehr ernst. Wir werden sehen, was daraus wird. Er hat uns nicht eingeschüchtert und schüchtert uns nicht ein. Wir haben starke Nerven. Das haben wir [schon] mehrfach bewiesen.

Wir können sagen, dass auf unserer Seite, also auf der Seite derer, die keinen Krieg wollen, mehr Menschen stehen als auf der Seite derer, die Krieg wollen. So ist die Lage sogar in Amerika, wo das Volk ebenfalls keinen Krieg will.

Ich möchte bemerken, sagt N. S. Chruschtschow, dass die Liquidierung des „Kalten Krieges“ am nützlichsten ist, und darum sollte man sich bemühen. Daher will ich Ihnen sagen, dass keines der Worte, die ich in diesem Gespräch geäußert habe, als Instrument für ein weiteres Anfachen des „Kalten Krieges“ verwendet werden sollte. Schließlich ist der „Kalte Krieg“ keineswegs ein Segen für die Völker.

Ich würde es für eine große Auszeichnung - für mich persönlich und für mein Volk - halten, wenn zwischen unseren Ländern friedliche und freundschaftliche

106 Wie nach wiederholten Äußerungen Chruščëvs bei anderen Gelegenheiten zu vermuten ist, dachte er daran, dass Eisenhower als Oberbefehlshaber der amerikanischen und britischen Truppen in Mitteleuropa in der Schlussphase der Kämpfe auf den Vorstoß nach Berlin verzichtete, um, wie er der sowjetischen Seite mitteilte, der Roten Armee die Eroberung der deutschen Hauptstadt zu überlassen. 
Beziehungen hergestellt werden könnten. Schließlich wollen das alle einfachen Leute unseres Landes wie auch die einfachen Leute der USA.

Unser Gespräch hat sich lange hingezogen. Ich möchte Ihnen dafür danken, weil ich in gewissem Maße die echte Stimme Amerikas gehört habe. Nicht jene „Stimme Amerikas“, die per Radio verbreitet wird107 - auf die höre weder ich noch andere Sowjetmenschen. Ich denke, dass unser Gespräch Nutzen bringen wird.

$M c D$ uffie dankt N. S. Chruschtschow für das aufrichtige und gute Gespräch und sagt, er werde sich bemühen, alles, was N. S. Chruschtschow gesagt habe, an das amerikanische Volk weiterzugeben.

Er äußert den Wunsch, seine Gesprächsniederschrift mit der Niederschrift abzugleichen, die während des Gespräches [von sowjetischer Seite] angefertigt wurde.

Chruschtschow bringt sein Einverständnis zum Ausdruck.

$M c D$ uffie teilt mit, dass er mit dem Erlernen der russischen Sprache begonnen hat. Sich der Karte zuwendend, die an der Wand hängt, sagt er: Es beunruhigt mich, dass auf Ihrer Karte die Vereinigten Staaten von Amerika in so kleinem Maßstab dargestellt ist. Auf unserer Karte sieht das ganz anders aus.

Chruschtschow: Hier ist nichts entstellt worden, sagt N. S. Chruschtschow. Alles ist im gleichen Maßstab dargestellt.

McDuffie: Auf unseren Karten sind die Vereinigten Staaten von Amerika in die Mitte gerückt.

Chruschtschow: Es wäre nicht zu verstehen, wenn auf der Karte, die bei mir hängt, die USA in der Mitte wären. Sie würden sagen, Chruschtschow führt etwas im Schilde. Der Maßstab auf der Karte ist jedoch für unser Land und für die anderen Länder gleich.

McDuffie bedankt sich nochmals für das Gespräch.

Aufgezeichnet von I. Bubnow

Übersetzt aus dem russischen Originaltext

RGANI, fond 52, opis‘ 1 , delo 578, B1.51-73

(Gesamter Text: B1. 43-72)

107 Die von der Information Agency der USA betriebene Rundfunkstation Voice of America wandte sich an das Ausland. Vor allem die Schlagermusik verschaffte ihr weltweit ein großes Publikum, das sich dann oft auch die - in der jeweiligen Landessprache gesendeten - Nachrichten anhörte. 